\title{
PERIODIC SOLUTIONS OF LINEAR DIFFERENTIAL EQUATIONS
}

BY W. B. FITE

The present article contains a generalization and a correction of certain results given by the writer in an earlier article under the same title.* Consider the equation

$$
L(y) \equiv p_{0} y^{(n)}+p_{1} y^{(n-1)}+\cdots+p_{n} y=0,
$$

in which the coefficients are one-valued and of period $l$, and $p_{0} \neq 0$ for any real value of $x$. If $y_{1}, y_{2}, y_{3}, \cdots, y_{n}$ form a funda mental system of solutions, then

where

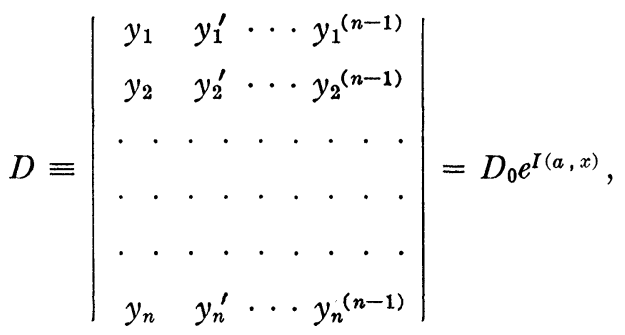

$$
I(a, x)=-\int_{a}^{x} \frac{p_{1}}{p_{0}} d x
$$

and where $D_{0}(\neq 0)$ is the value of $D$ for $x=a$. Since the coefficients of (1) are of period $l, y_{i}(x+l),(i=1,2, \cdots, n)$, form a fundamental system of solutions, and therefore

$$
y_{i}(x+l)=\sum_{j=1}^{n} a_{i j} y_{j} .
$$

The characteristic equation of the substitution (2) is

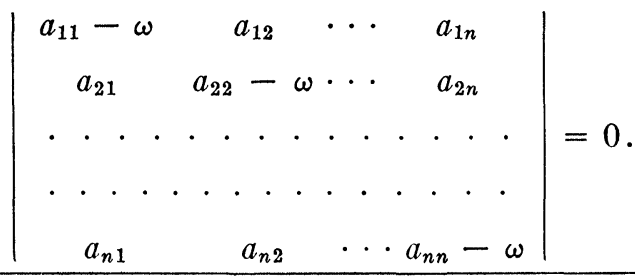

* Fite, Annals of Mathematics, (2), vol. 28 (1926), pp. 59-64. 
If

$$
\left(\omega-\omega_{1}\right)^{\mu_{1}}, \quad\left(\omega-\omega_{2}\right)^{\mu_{2}}, \quad \cdots, \quad\left(\omega-\omega_{k}\right)^{\mu_{k}},
$$

where $\mu_{1}+\mu_{2}+\cdots+\mu_{k}=n$, are the elementary divisors of the left member of (3), there is a canonical fundamental system of solutions $y_{i, i},\left(i=1,2, \cdots, k ; j=1,2, \cdots, \mu_{i}\right)$, such that ${ }^{*}$

$$
\begin{aligned}
& y_{i, 1}(x+l)=\omega_{i} y_{i, 1}, \\
& y_{i, 2}(x+l)=y_{i, 1}+\omega_{i} y_{i, 2}, \\
& . . \\
& \text {. . . . . . . . . } \\
& y_{i, \mu_{i}}(x+l)=y_{i, \mu_{i}-1}+\omega_{i} y_{i, \mu_{i}} .
\end{aligned}
$$

In terms of these solutions

$D(x+l)=$

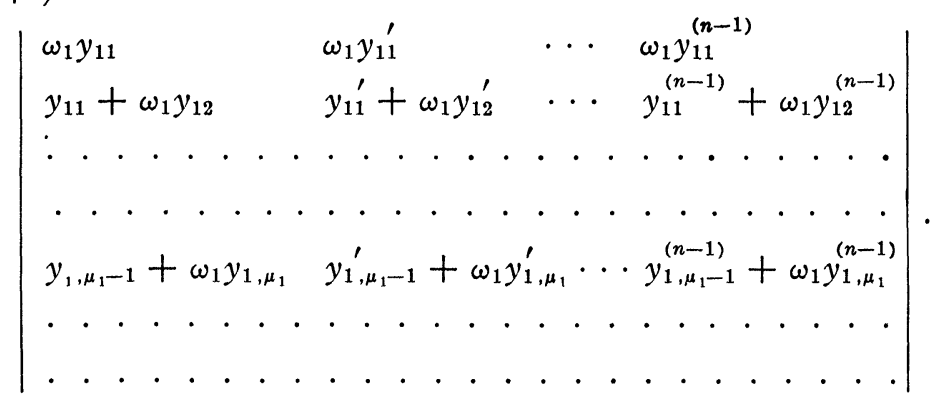

The first $\mu_{1}$ rows are here indicated in detail. The remaining rows are formed similarly in sets of $\mu_{2}, \mu_{3}, \cdots, \mu_{k}$ each corresponding to the respective elementary divisors. It readily follows that

$$
D(x+l)=\omega_{1}^{\mu_{1}} \omega_{2}^{\mu_{2}} \cdots \omega_{k}^{\mu_{k}} D(x)
$$

But on the other hand

$$
D(x+l)=D_{0} e^{I(a, x+l)}=D_{0} e^{I(a, x)+I(x, x+l)}=e^{I(x, x+l)},
$$

where, as above,

$$
I(u, v)=-\int_{u}^{v} \frac{p_{1}}{p_{0}} d x .
$$

* See, for example, Horn, Gewöhnliche Differentialgleichungen beliebiger Ordnung, p. 85. 
Hence

$$
\omega_{1}^{\mu_{1}} \omega_{2}^{\mu_{2}} \cdots \omega_{k}^{\mu_{k}}=e^{I(x, x+l)}
$$

If there is a solution of (1) of period $l$, at least one of the $\omega$ 's is 1 ; and conversely if at least one of the $\omega$ 's is 1 there is a solution of (1) of period $l$. If all of the solutions of (1) are of period $l$, every $\omega=1$ and therefore

$$
e^{I(x, x+l)}=1, \text { or } \int_{x}^{x+l} \frac{p_{1}}{p_{0}} d x=0 .
$$

But if every $\omega=1$ not every solution is necessarily of period $l$. For example, for certain values of $a$ the Mathieu equation

$$
y^{\prime \prime}+(a-2 b \cos 2 x) y=0
$$

has a solution of period $\pi$. We can take $l=\pi$. Moreover $p_{0} \equiv 1$ and $p_{1} \equiv 0$. Hence

$$
\int_{x}^{x+l} \frac{p_{1}}{p_{0}} d x=0 .
$$

That is, the product of the $\omega$ 's is 1 and one of them is 1 . Hence the other one is also 1 . But not every solution is of period* $\pi$.

Consider now the adjoint of (1); namely

$$
\bar{L}(z)=0 \text {. }
$$

The functions

$$
z_{i j}=\frac{1}{p_{0}} \frac{\partial \log D}{\partial y_{i j}^{(n-1)}}
$$

form a fundamental set of solutions of $\dagger$ (5). It follows from relations (6) that when $x$ is changed to $x+l$ the $z$ 's are transformed contravariantly to the $y$ 's. The determinant of this transformation is made up as follows.

Corresponding to the $i$ th elementary divisor of (3) there is a minor of this determinant of the form

* Ince, Ordinary Differential Equations, p. 177.

$\dagger$ Darboux, Théorie des Surfaces, vol. 2, p. 102. 


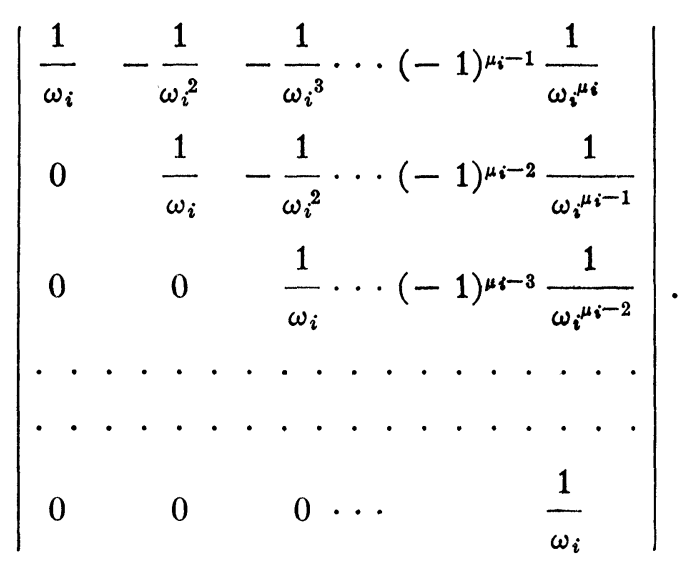

The principal diagonal of this minor lies on the principal diagonal of the determinant, and every element of the determinant that does not belong to one of these minors is zero.

The roots of the characteristic equation of this transformation on the $z$ 's are the reciprocals of the roots of the corresponding equation for the $y$ 's. Moreover, the elementary divisors of the left member of the characteristic equation relating to the $z$ 's are the same as those for the left member of (3) with the exception that $\omega-1 / \omega_{i}$ takes the place of $\omega-\omega_{i}$. Hence (1) and its adjoint (5) have the same number of linearly independent solutions of period* $l$. It follows that if $L(y)$ is an exact derivative, the equation $L(y)=0$ has a solution of period $l$, since $\bar{L}(z)=0$ has a constant for a solution, and this is of period $l$.

If now (1) is self-adjoint the equation (3) is a reciprocal equation, and if $n$ is odd there must be an odd number of roots that are their own reciprocals; that is, an odd number of roots must be \pm 1 . But if an odd number of these were equal to -1 , the product of all the roots would be -1 , since the other roots that are different from 1 occur in pairs of reciprocals. On the other hand, since (1) is by supposition self-adjoint, $p_{1}=n p_{0}^{\prime} / 2$, and

$$
\left.-\int_{x}^{x+l} \frac{p_{1}}{p_{0}} d x=-\frac{n}{2} \int_{x}^{x+l} \frac{p_{0}^{\prime}}{p_{0}} d x=-\frac{n}{2} \log p_{0}\right]_{x}^{x+l}=0 .
$$

That is, by (4), the product of the roots of the characteristic equation (3) must be +1 . Hence an odd number of these roots

* See Fite, loc. cit., p. 61. 
are equal to 1 and equation (1) has at least one solution of period $l$.

A similar analysis applies if we pass to the complex plane and require merely that the coefficients be one-valued in the neighborhood of $z=z_{0}$ and that $p_{0}$ have a pole of order $j$ at this point $(j \geqq 0)$. If $(1)$ is self-adjoint the product of the roots of the characteristic equation of the transformation on a fundamental system of solutions due to a circuit around $z=z_{0}$ is equal to

$$
e^{I_{C}}=e^{n j \pi i}= \pm 1, \quad I_{C}=-\frac{n}{2} \int_{C} \frac{p_{0}^{\prime}}{p_{0}} d x
$$

according as $n j$ is even or odd. If $n$ is odd and $j$ is even, $n j$ is even and an odd number of the roots of the characteristic equation are equal to 1 . Hence there is at least one solution that is one-valued in the neighborhood of $z=z_{0}$. But if $n$ and $j$ are both odd, an odd number of the roots of the characteristic equation are equal to -1 , and there is at least one solution that is twovalued in the neighborhood of $z=z_{0}$.

If in (1) we again assume that the coefficients are real and of period $l$ and put $y=e^{m x} t, t$ will be a solution of the equation

$$
\begin{aligned}
& p_{0} t^{(n)}+\left(n m p_{0}+p_{1}\right) t^{(n-1)}+\cdots \\
& \quad+\left(m^{n} p_{0}+m^{n-1} p_{1}+\cdots+m p_{n-1}+p_{n}\right) t=0 .
\end{aligned}
$$

If now $t$ is a solution of ( 7$)$ of period $l, y$ is a solution of (1) such that

$$
y(x+l)=e^{m l} y(x) .
$$

Hence (7) cannot have solutions of period $l$ for more than $r$ real values of $m$, where $r$ is the number of distinct positive roots of (3). Nor can it have semi-periodic solutions with a given real multiplier $\sigma$ for more than $r$ real values of $m$, where $r$ is the number of distinct roots of this characteristic equation that have the same $\operatorname{sign}$ as $\sigma$.

Suppose that

$$
L(y)=\phi(x),
$$

where $\phi(x)$ is not identically zero and is of period $l$, has a solution $y$ of period $l$. This assumption is certainly realized if (1) has no solution of period $l$ other than* $y \equiv 0$. If $(1)$ has $r(r>0)$ linearly

* Fite, loc. cit., p. 60, Theorem I. 
independent solutions of period $l$, equation (5) has the same number. We represent them by $z_{1}, z_{2}, \cdots, z_{r}$. If now in the Lagrange identity

$$
\int[z L(y)-y \bar{L}(z)] d x=\psi(y, z)
$$

we put for $y$ a solution of (8) of period $l$ and $z_{i}$ for $z$, we get

$$
\int z_{i} \phi(x) d x=\psi\left(y, z_{i}\right) .
$$

Now $\psi\left(y, z_{i}\right)$ is a polynomial in $y$ and $z_{i}$ and their derivatives together with the coefficients of $L(y)$ and their derivatives. It is therefore of period $l$ and $z_{i} \phi(x)$ is the derivative of a function of this period. This means that in order that (8) have a solution of period $l$ it is necessary that $z \phi(x)$ be the derivative of a function of this period in case $z$ is any solution of (5) of this period.

We consider now whether this condition is sufficient. If $t$ is a solution of the adjoint of the equation $L^{\prime}(y)=0, t L^{\prime}(y)$ is an exact derivative, as is also $t L^{\prime}(y)+t^{\prime} L(y)$. Hence $t^{\prime} L(y)$ is an exact derivative and $t^{\prime}$ is a solution of $\bar{L}(y)=0$. Moreover, if $t$ is a function such that $t^{\prime} L(y)$ is an exact derivative, then $t L^{\prime}(y)$ is an exact derivative and $t$ is a solution of the adjoint of $L^{\prime}(y)$ $=0$. Hence this adjoint is $\bar{L}\left(t^{\prime}\right)=0$.

The solutions of $L(y)=0$ and $L(y)=c$, where $c$ is any constant $\neq 0$, are solutions of $L^{\prime}(y)=0$ and every solution of this last equation is a solution of one or the other of the two preceding ones. If, then, $L(y)=0$ has $r(r>0)$ linearly independent solutions of period $l$, and no more, and $L(y)=c$ has no such solution, $L^{\prime}(y)=0$ has only $r$ linearly independent solutions of this kind, and its adjoint has the same number. If now every one of the $r$ linearly independent solutions $z_{1}, z_{2}, \cdots, z_{r}$ of $\bar{L}(y)=0$ of period $l$ were the derivative of a function of this period, that is, if $t_{i}=\int z_{i} d x,(i=1,2, \cdots, r)$, were of period $l$, then the $t_{i}$ 's together with $t_{n+1} \equiv 1$ would form a system of $r+1$ linearly independent solutions of the adjoint of $L^{\prime}(y)=0$ of period $l$. But we have just seen that this adjoint has only $r$ linearly independent solutions of this period in case $L(y)=0$ has only $r$ linearly independent solutions of this kind and $L(y)=c$ has none. If $L(y)=0$ has no solution of period $l$ other than $y \equiv 0$, the condition in 
question is obviously satisfied and, as we have seen, $L(y)=c$ has a solution of this period. The condition is therefore sufficient in case $\phi(x)$ is a constant.

Suppose now that $\phi(x)$ is a non-constant function of period $l$. The solutions of (8) are also solutions of the equation

$$
\frac{L(y)}{\phi(x)}=1,
$$

for all values of $x$ for which $\phi(x) \neq 0$, and the coefficients of (9) are of period $l$. Moreover, $z_{i} \phi(x),(i=1,2, \cdots, n)$, form a linearly independent set of solutions of the adjoint of the equation $L(y) / \phi(x)=0$, and $z_{1} \phi(x), z_{2} \phi(x), \cdots, z_{r} \phi(x)$ are all of these that are of period $l$. Therefore a sufficient condition that (8) have a solution of period $l$ is that $z_{i} \phi(x),(i=1,2, \cdots, r)$, be the derivatives of functions of period $l$, and this condition is necessary. This is equivalent to the condition $\int_{x}^{x+l} z_{i} \phi(x) d x=0,(i=1$, $2, \cdots, r)$. We have therefore the following theorem.

THEOREM. If $p_{0}(x) \neq 0$ and $\phi(x) \neq 0$, while $\phi(x)$ and the coefficients of $L(y)$ are of period $l$, a necessary and sufficient condition that $L(y)=\phi(x)$ have a solution of period $l$ is that $\int_{x}^{x+l} z \phi(x) d x=0$ when $z$ is any solution of $\bar{L}(y)=0$ of period $l$.

The sufficient condition given in Theorem 2 of the article referred to in the first paragraph is obviously incorrect, as may be seen by considering the equation

$$
y^{\mathrm{iv}}+y^{\prime \prime}=1 \text {. }
$$

The right member and the coefficients in the left member are of period $2 \pi$. Moreover $y=\sin x$ is a solution of period $2 \pi$ of the corresponding homogeneous equation, which is self-adjoint, and $\int_{x}^{x+2 \pi} \sin x d x=0$, while not every solution of the homogeneous equation is of period $2 \pi$. And yet the original equation has no periodic solution, since if $y$ were such a solution we should have $y^{\prime \prime \prime}+y^{\prime}=x+c$. But this is impossible since the left member would be periodic while the right member is not. This is in conformity with the conditions of the present theorem since $z=1$ is a solution of period $2 \pi$ of the adjoint of the homogeneous equation and $\int_{x}^{x+2 \pi} d x \neq 0$.

Columbia University 